\title{
Modelo integrador para la formación de profesionales de la comunicación en entornos virtuales: preparando emprendedores
}

\author{
Juan Carlos Marcos Recio \\ Julio Alcolado Santos *
}

Artículo recibido:

9 de mayo de 2013.

Artículo aceptado:

27 de febrero de 2014.

\section{RESUMEN}

Las Nuevas Tecnologías, y especialmente Internet, han revolucionado el mundo de los medios de comunicación. Los lectores demandan nuevos formatos para la información y el sector profesionales con las habilidades que las nuevas circunstancias exigen. En este estudio se pretende descubrir y presentar un modelo integrador para la educación superior que funcione en los entornos virtuales de aprendizaje y que sirva de referente para otros docentes del área de comunicación. El modelo propone la realización de prácticas reales desarrolladas en entornos profesionales, donde se muestra cómo la nueva manera de enseñar y aprender es parte de un mismo lugar en el que alumnos y docen-

Ambos autores pertenecen a la Universidad Complutense de Madrid, España jmarcos@ucm.es; jalcolad@ucm.es

INVESTIGACIÓN BIBLIOTECOLÓGICA, Vol. 28, Núm.64, septiembre/diciembre, 2014 , México, ISSN: 0187-358X. pp. 75-100 
tes comparten iniciativas, apoyos, recursos, diseños
formativos, prácticas y sobre todo experiencias.

Palabras clave: Comunicación; Periodismo; Educación Virtual; Metodología; Emprendimiento.

\begin{abstract}
Integrative model for training communications professional in virtual environments: creating entrepreneurs Juan-Carlos Marcos-Recio and Julio Alcolado-Santos

New Technologies, especially the Internet, have revolutionized the mass media world. Readers demand new formats for information and industries needs professionals who have skills adapted to the new realities. This study aims to present an integrative model for higher education for teaching in virtual learning environments, and attempts to serve as a benchmark for other teachers in the field of communication. The integrative model proposes real training developed in professional environments. This model demonstrates a new way of teaching and learning, where students and teachers share initiatives, resources, training designs and experiences in the same place.
\end{abstract}

Keywords: Communication; Journalism; Virtual Education; Methodology; Entrepreneurship.

\title{
INTRODUCCIÓN
}

Tunca antes los medios de comunicación habían experimentado un cam1 bio tan significativo. Pasada la primera década del siglo XXI, la segunda nos adentró en una transformación global que afecta al mundo empresarial, social y personal. La información fluye de tal manera que los ciudadanos se han sentido protagonistas y colaboradores a través de blogs, redes sociales y entornos virtuales de participación. Son propuestas en las que el proceso informativo rompe su esquema tradicional y convierte a la información en una parte importante de la actividad de los ciudadanos. Ya no importa tanto la reflexión profunda de la información. Se requieren cada vez menos datos 
y una forma de comunicar dinámica, porque el usuario de los medios tiene acceso a muchas y variadas fuentes de información.

Este cambio de paradigma es lo que Carr analiza en primera persona en Superficiales. ¿Qué está baciendo Internet con nuestras mentes?: "En algún momento de 2007, [...] No era sólo que muchos de mis hábitos y rutinas estaban transformándose mientras me acomodaba cada vez más y me hacía dependiente de las páginas y servicios de la Red. El modo mismo en que mi cerebro funcionaba parecía estar cambiando. Fue entonces cuando empecé a preocuparme sobre mi incapacidad para prestar atención a una sola cosa durante más de dos minutos. [...] Estaba hambriento, exigía ser alimentado en la manera que lo alimentaba la Red, y cuanto más comía, más hambre tenía" (Carr, 2011: 29). Es en este nuevo sistema en el que se envuelve la nueva educación, el que evidencia un nuevo cambio de metodología en el que los alumnos son capaces de hacer/actuar en varios frentes a la vez. En los sistemas tradicionales, el docente trabajaba en su sesión de clase con una o varias ideas de forma lineal; ahora los alumnos son capaces de comprender esas ideas y de ampliarlas con enlaces y videos. Su atención, lejos de perderse, se concentra cuando se les ofrecen herramientas capaces de mostrar un concepto de varias maneras.

Si el cambio es de tal magnitud en los medios y también en el resto de la sociedad, ¿qué va a ocurrir en la formación de estos profesionales? ¿Qué tipo de estudios merecen ser aplicados en las facultades que imparten grados de comunicación en los tres frentes: periodismo, publicidad y relaciones públicas y comunicación audiovisual? ¿Será precisa una formación integradora que aporte excelentes comunicadores a la sociedad? Son muchas las preguntas y las inquietudes que tienen los formadores en comunicación, pero tienen cada vez más claro que la formación que emplea entornos virtuales es más constructiva y permite una mejora en los resultados académicos.

Es propósito de este trabajo descubrir y presentar un modelo integrador que funcione en los entornos virtuales de aprendizaje y que sirva de referente para otros docentes del área de comunicación. Se trata de mostrar cómo la nueva manera de enseñar y aprender es parte de un mismo lugar en el que alumnos y docentes comparten iniciativas, apoyos, recursos, diseños formativos, prácticas y, sobre todo, experiencias.

Pasada la euforia de las competencias, por otra parte necesarias, es tiempo de concretar resultados válidos y de aplicarlos en la educación superior. Si algo es aceptablemente bueno en estos entornos es la capacidad de adaptación y uso en otras instituciones educativas. Los avances y también los retrocesos, los ensayos y pruebas ya están dejando paso a algunos primeros resultados favorables en universidades que antes dudaban, pero ahora ven con 
buenos ojos la aplicación de procesos educativos basados en entornos virtuales. Ya ni siquiera las grandes universidades con siglos de historia presencial y con grandes maestros se alejan de esta nueva realidad. Los tiempos de la imprenta se fueron para los medios y llegaron los digitales. Los tiempos del libro y el saber acumulado se marcharon y en su lugar aparecen cada vez más docentes con textos virtuales colaborativos, con prácticas conjuntas entre varios profesores, con blogs y herramientas de los alumnos que aportan ideas y sobre todo con un apoyo importante por parte de los docentes que tardaron, por relevo generacional, en entrar en los campus virtuales para mostrar su saber y adaptarse.

Esta iniciativa colaborativa ha tenido su momento álgido en los Open Educational Resources (OER), cuya traducción es Recursos Educativos Abiertos (REA), término acuñado por la UNESCO en 2002. No obstante, el movimiento es mucho más conocido como OCW (Open Course Ware o "contenidos abiertos") promovido en abril de 2001 por el Instituto Tecnológico de Massachusetts (MIT) para la publicación de materiales docentes bajo licencia Creative Commons, que permite el uso y la reutilización de los contenidos.

Desde 2005 existe el OpenCourseWare Consortium, como consecuencia de unir todos los proyectos OCW que surgieron en diferentes partes del mundo y cuyo objetivo es conseguir mayor difusión, coordinación y ser un foro de intercambio entre organizaciones para el fomento y la estrategia futura de la propia organización. Es en definitiva una comunidad a nivel mundial con presencia en 44 países del mundo, donde participan cientos de instituciones de educación superior y organizaciones asociadas.

También Europa apuesta por cursos de este tipo, muy variados en contenidos, en los que cada socio ofrece los cursos en su propia plataforma de aprendizaje. Se puede elegir entre las 11 lenguas de los socios y el árabe. Es un acuerdo de 11 países, entre ellos España, que ofrecen 40 cursos masivos abiertos en línea (Massive Open Online Courses-MOOC) con el fin de modernizar los sistemas de educación superior:

La European Association of Distance Teaching Universities (EADTU, Asociación Europea de Universidades de Educación a Distancia), que agrupa principalmente a universidades abiertas de Francia, Italia, Lituania, los Países Bajos, Portugal, Eslovaquia, España, Reino Unido y Rusia, Turquía e Israel, ha puesto en marcha la primera iniciativa paneuropea de cursos masivos abiertos online (Massive Open Online Courses-MOOC), con el respaldo de la Comisión Europea. (Ingenieros.es, 2013: s. pág.) 


\section{COMUNICACIÓN Y EDUCACIÓN EN TIEMPOS DE LAS REDES SOCIALES}

Desde el comienzo de la humanidad la comunicación ha sido el elemento que ha sustentado la estructura social. Sin comunicación la sociedad sería diferente. Si se une comunicación y educación, el binomio completo servirá para que la sociedad avance hacia nuevos descubrimientos. Este paradigma educativo, que funcionó durante siglos, tiene los días contados. La velocidad con la que circula la información en los medios de comunicación digitales y la respuesta de los usuarios a través de las redes sociales muestran otra manera de enfrentar el mundo de la enseñanza.

Siendo realistas, los métodos que ponen al estudiante/alumno frente al profesor están perdiendo fuerza. Quienes piensan que colocar al mismo nivel a ambos es un acierto tampoco han conseguido mejores resultados. Es tiempo entonces de formular una propuesta integradora en la que los docentes compartan sus recursos con otros de su misma área temática y los alumnos tengan a su disposición más material y mejores propuestas para entenderlo. Hasta ahora los docentes apenas compartían información sobre sus propuestas educativas. Sus prácticas se presentaban a título individual y el estudiante no tenía nunca una visión integral de la propuesta de dicho profesor. Con el uso de la tecnología, algunos profesores están configurando casos prácticos en los que intervienen diversas materias/asignaturas y cada docente evalúa una parte de la misma. Si en la materia Historia de la Publicidad se requiere trabajar sobre publicitarios que contribuyeron a mejorar esta disciplina, el uso de las fuentes de información debe estar presente. En este sentido, otra materia como Documentación Publicitaria puede facilitar el uso de dichas fuentes. $Y$ así es posible asociar muchos casos prácticos que permitirán al alumno tener una visión global de sus estudios y no una tan particular, como viene sucediendo.

La comunicación es un acto que transmite información desde un emisor a un receptor mediante el uso de un canal. La educación es también un acto formativo en el que la información está sustentada por la explicación del docente. Sin embargo, en tiempos de las redes sociales, junto a esa explicación se pueden/deben aportar otras en las que el alumno haya trabajado previamente, con lo que el grupo saldrá reforzado y con varias maneras de entender una propuesta. Esto incluye los aprendizajes complejos, donde las herramientas actuales permiten una mejora considerable tal y como se señala en el Informe Horizon 2010:

Al integrar múltiples formas de expresión y de comunicación multimedia, los medios sociales se adaptan a distintos estilos de aprendizaje y a la vez son óptimos 
para facilitar el desarrollo de aprendizajes complejos, lo que promueve el desarro-

llo de comunidades de aprendizaje. (García et al., 2010: 18)

Estas comunidades son las que están formulando/exigiendo un modelo integrador, pues suponen una mejora en los resultados educativos y ponen a disposición de los estudiantes no una sino varias ideas desarrolladas. $\mathrm{Na}$ die duda que la educación emplea cada vez más herramientas, imágenes de video, clips de audio, podcasts y presentaciones multimedia de elaboración propia o de otros autores, activos desde el Campus Virtual de la institución o de lugares de uso común para profesores y/o alumnos, tipo YouTube, Flickr, Twitter, Delicious, Slideshare, entre otros. Pero ahora hay una función nueva e importante. El alumno es proactivo y a la vez prosumidor: crea y consume la información de las redes sociales. En apenas dos años ha pasado de consultar (usuario inactivo) a producir, ordenar, compartir, comentar y valorar (usuario activo) las propuestas que circulan en las redes sobre una materia, o los materiales que el docente de una determinada materia ha colocado en esos sitios. Por tanto, la principal diferencia entre los modelos educativos de antes y los que se desarrollan en las redes sociales está en la creatividad; en la participación más activa de los alumnos en el contenido docente.

Pero, de todos estos soportes, ¿cuáles están siendo más utilizados por los docentes y alumnos? Los dispositivos móviles, videoconferencias y chats en línea son los ejemplos más evidentes de estas tecnologías: permiten la comunicación instantánea.

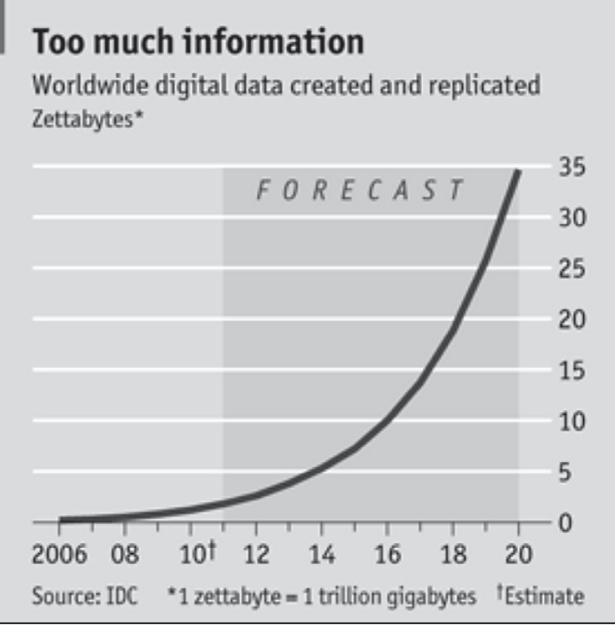

Figura 1. Evolución de la tecnología hasta 2020. Fuente: The Economist(2011). 
Resulta fácil ver cómo los servicios destinados a obtener datos son cada vez más abundantes. Son una razón por la que IDC, una firma de investigación de mercado, predice que el "universo digital", la cantidad de información digital creada y reproducida en un año, aumentará a 35 zettabytes para el año 2020 desde menos de 1 zettabyte en 2009 (véase Figura 1), donde 1 zettabyte es de 1 billón de gigabytes, o el equivalente a 250 billones de DVD.

Pero estas herramientas también harán que las fronteras de una empresa sean cada vez más porosas. "WikiLeaks es sólo un reflejo del problema en el que cada vez más datos se producen y pueden filtrarse", dice John Mancini, presidente de la AIIM (Assocciation for Information and Image Management), una organización dedicada a mejorar la gestión de la información (The Economist, 2011).

Otras dos novedades también hacen de cortafuegos de las empresas digitales. Una de ellas es la externalización: los contratistas a menudo necesitan ser conectados a los sistemas informáticos de sus clientes; la otra está constituida por una especie de artilugios empleados de manera propia. Los más jóvenes, sobre todo, quienes están en sintonía con la tecnología fácil de usar del consumidor, desean traer su propio equipo de trabajo. "No me gusta usar una BlackBerry corporativa porque es aburrido”, explica Mancini (The Economist, 2011: s. pág.).

\section{MODELO INTEGRADOR PARA LA COMUNICACIÓN: UNA ADAPTACIÓN ESCOLAR A LA UNIVERSIDAD}

No son tiempos de milagros. Son, si acaso, de experimentación. Lo que antes no funcionaba ahora se puede mejorar, pero no resolver. Mi experiencia como docente en grupos presenciales numerosos (más de 150 alumnos) me aseguraba resultados más bien pobres. En esos grupos sólo un $25 \%$ de los estudiantes eran capaces de seguir la dinámica del profesor. Y si además éste centraba su trabajo teórico en explicar ideas fundamentales de la materia y los estudiantes apenas seguían el proceso de copiar notas, sólo quedaba una solución: retrasar la dinámica del grupo, es decir, ir más despacio y trabajar con menos materia. Pero ¿qué pasa con el grupo de alumnos que son capaces de avanzar y quieren que se les explique toda la materia? Hasta ahora pocos profesores habían resuelto esta situación, pero la llegada de la tecnología y los entornos virtuales formales e informales que se han desarrollado les ha permitido lograrlo. Lo que algunos autores llaman escuela híbrida, una mezcla entre educación tradicional apoyada por la tecnología, está sirviendo de referencia para un nuevo método de enseñanza en el que cada estudiante lleva un ritmo diferente y no frena al grupo. 
Algunos expertos en Entornos Virtuales de Aprendizaje (EVA) dirán que no es nada nuevo, pero sí hay un matiz importante ya que no sólo se explica en la teoría, sino que se hace de manera práctica. El principal objetivo de estas escuelas híbridas es conseguir que cada estudiante disponga de una educación personalizada a lo largo de todas las horas lectivas. Si se vuelve la vista atrás, ésta fue la fórmula empleada durante muchos siglos en Europa. Luego, tras la Revolución Industrial y la llegada masiva de alumnos a las aulas de las universidades europeas a partir de la Segunda Guerra Mundial, el sistema cambió.

Existe un tipo de educación escolar que se está aplicando en Estados Unidos, aunque todavía de forma experimental. El programa se llama School of One (http://www.schoolofone.org) y ha sido reconocido como una de las ideas más innovadoras por la revista Time. Se inició en el verano de 2009 en una escuela secundaria de Manhattan. El esfuerzo se hace para cada individuo, no para el colectivo. La idea fundamental: "Dar a cada alumno lo que necesita, no pensar en las necesidades de toda la clase". En definitiva, hacer una ruta para cada estudiante.

Llegados aquí se plantea la siguiente cuestión: ¿se podría adaptar este modelo a la educación superior? ¿Se pueden proponer modelos similares para la universidad? ¿Es un modelo integrador o disgregador? ¿La suma de las voluntades de cada alumno y sus mejoras no hacen un grupo más activo y dinámico? ¿Pueden las universidades sostener financieramente un modelo de estas características? Buscando las respuestas a estas preguntas habremos avanzado significativamente en la evolución de la educación superior.

El método integrador, siendo de uso en la escuela primaria, se puede aplicar a la universidad. La propuesta es clara, pues cada estudiante debe disponer de herramientas que le permitan concretar sus necesidades educativas, personalizando de esta forma la enseñanza y utilizando la tecnología necesaria y precisa que las redes sociales facilitan. Probablemente sólo hay una manera de enseñar: el alumno espera obtener ideas, notas, apuntes; conocimiento, en definitiva. Lo que cambia es la forma de hacerlo, de ahí que tanto en comunicación como en periodismo y en publicidad los tiempos digitales requieren de una personalización de la educación y el uso de tecnologías para hacer prácticas reales, en las que el estudiante sea el protagonista, lo que luego le servirá como ejercicio práctico en su futuro profesional y laboral.

\section{Propuesta de modelo}

Este modelo docente toma como protagonista al estudiante y su entorno. Quizás muchos pedagogos insistan que en los sistemas tradicionales ya se ha- 
bían constatado fórmulas parecidas. El sistema educativo en el que se mueve logra que el alumno avance en función del ritmo y de las necesidades de cada uno. No se trata de excluir, sino de lograr que quien tenga más y mejores capacidades siga su formación en grupos diferentes y con niveles más avanzados. En otras palabras, se trata de romper con el modelo que coloca a los alumnos en un grupo único y en el que deben integrarse durante varios años de su vida docente, cuando esa relación en muchos casos es perjudicial para aquellos que quieren o pueden avanzar a otro ritmo. Todo esto se basa en el fomento del esfuerzo y la mejora continuada, que también motiva a los estudiantes que van menos avanzados y evita que se generen grupos de exclusión en la educación superior, tal como está ocurriendo en la Educación Secundaria española.

El modelo planteado es muy diferente al nuevo universo MOOC (Massive Open Online Course), Cursos en Línea Masivos y Abiertos que han sido popularizados por edX o Coursera. El objetivo fundamental de los MOOC es la liberación del conocimiento para que llegue a un público más amplio; es decir, que el conocimiento salga del entorno meramente universitario. Vendría a ser ésta una modalidad de educación abierta, que ofrece cursos de asignaturas universitarias de manera gratuita a través de plataformas educativas virtuales.

El modelo que planteamos es más bien una educación mixta, dentro de un ambiente universitario, que implique un concepto de aula definida, en el que se conozca el número de estudiantes y éste no sea excesivo, y donde exista un diseño y una estructura pedagógica claros, los conocimientos de los estudiantes sean bastante homogéneos y donde la personalización de la docencia sea uno de los elementos más importantes, y además se apoye en entornos virtuales formales y no formales, que permitan adquirir habilidades del mundo empresarial.

Otras iniciativas como la UNU (United Nations University, Universidad de las Naciones Unidas) o la Universidad del Pueblo (UoPeople), fundada en 2009 por el empresario educativo Shai Reshef, son modelos intermedios entre MOOC y School of One. En la UNU y en la UoPeople se encuentran similitudes con esta última, que tiene diseños académicos más elaborados, un concepto de aula e incluye un ambiente universitario, pero que siguen siendo modalidades educativas totalmente a distancia y de carácter masivo, donde encontramos demasiada heterogeneidad entre los estudiantes y no se permite la personalización de la docencia.

¿Cuáles son las características diferenciales de este modelo que proponemos? ¿Cómo se están aplicando? ¿Sirven para la educación superior? Son cuatro las propuestas para que el modelo funcione; a saber: 
1. Cada estudiante tiene diferentes necesidades. Cada estudiante llega a la sala de clases con un perfil único de habilidades, conocimientos e intereses. Por este motivo, los ritmos de trabajo deben ser diferentes. Esta misma situación se da en la educación superior, y más cuando se requiere el uso de computadoras personales, plataformas y otras tecnologías educativas. Se recomienda que los alumnos al entrar en un entorno virtual tomen un curso sobre tecnología para luego no estar descolgados del resto del grupo. Ese nivel tecnológico ya se requiere al momento de entrar en algunas universidades, como por ejemplo la Universidad de Guadalajara (UDG Virtual). En este sentido, lo más importante sería impartir ese nivel en la propia universidad, como hacen las nuevas universidades privadas surgidas en España (especialmente UEM -Universidad Europea de Madrid-, UDIMA -Universidad a Distancia de Madrid-, entre otras) y las escuelas de negocios (como IE -Instituto de Empresas-, IESE -Instituto de Estudios Superiores de la Empresa de la Universidad de Navarra-, ESADE -Escuela Superior de Administración y Dirección de Empresas-, ESICC -Escuela Superior de Gestión Comercial y Marketing- y EOI -Escuela de Organización Industrial-).

2. En las clases tradicionales no es posible enseñar de forma personalizada atendiendo a las necesidades e intereses de cada alumno. En consecuencia, se requiere implementar un plan individualizado que satisfaga las necesidades de cada estudiante y también la propia formación de los docentes, como sucede en las nuevas universidades privadas españolas. Sin embargo, en los entornos virtuales formales y no formales sí resulta más fácil hacer un seguimiento personalizado del alumno, apoyándonos en la tecnología.

3. Se dispone de una sofisticada tecnología que permite dar a cada alumno los recursos que precisa. Ésta es una de sus grandes aportaciones: usa la tecnología y la adapta a cada uno de los niveles de alumnos que haya en el aula (Figura 2). Se requiere del estudiante una implicación con el mundo tecnológico para que las herramientas facilitadas no le impidan el avance.

4. Hay que reinventar tiempos y espacios. Para hacer posible la personalización, School of One trabaja en grandes espacios que permiten diferentes formas de aprender al mismo tiempo; algunos estudiantes trabajan con los maestros, otros con los profesores más avanzados; se puede hacer un trabajo independiente con libros y ordenadores y algunos lo hacen también en pequeños grupos. La asistencia de múltiples habilidades permite enseñar en diferentes partes del espacio al 
mismo tiempo y cada estudiante es libre de trabajar a su ritmo (Figura 3). Se pide un cambio de mentalidad en los docentes, puesto que en este modelo concreto los alumnos se adaptan desde sus primeros días de clase a esta forma de estudio, con horarios más versátiles.

El problema se plantea cuando los estudiantes llegan a los estudios superiores y están habituados a una metodología diferente, con un ritmo adaptado para un grupo concreto y con escasa actividad práctica. El tiempo y el espacio cobran una nueva vida para los alumnos, pero también para los docentes, que toman las lecciones de fondos educativos ya existentes y siguen avanzando a su ritmo.

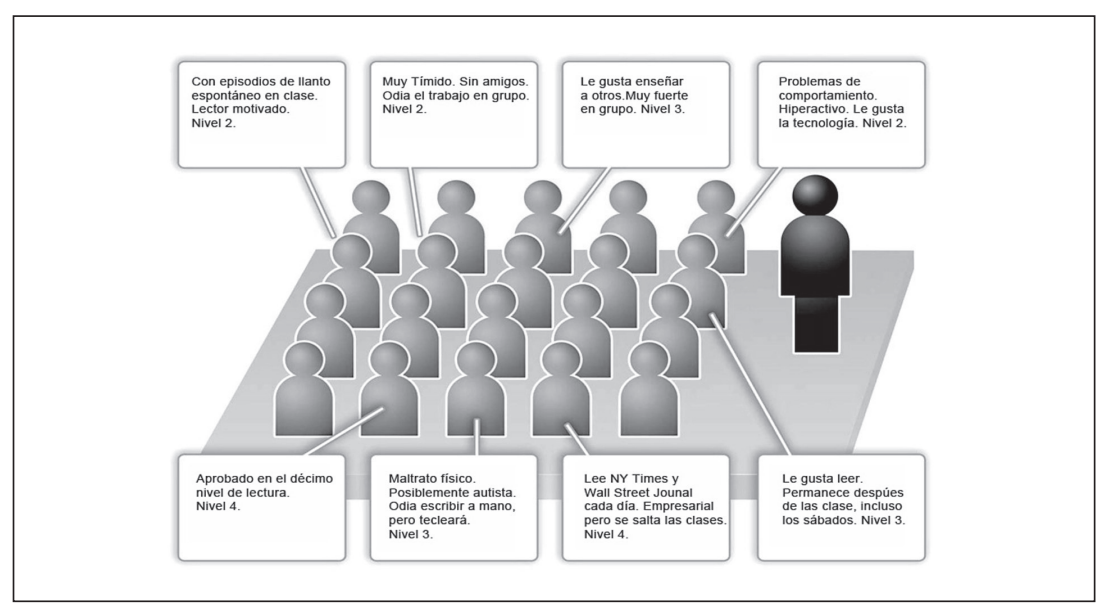

Figura 2. Distribución de alumnos en un aula con diferente niveles. Fuente: School of One.

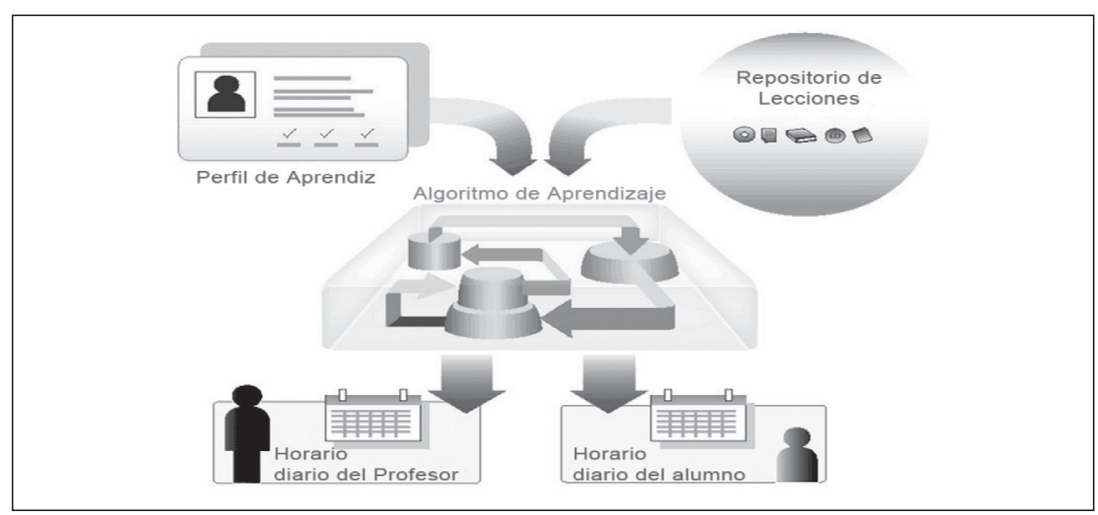

Figura 3. Avanzar en un tiempo y espacio diferentes a los actuales. Fuente: School of One. 


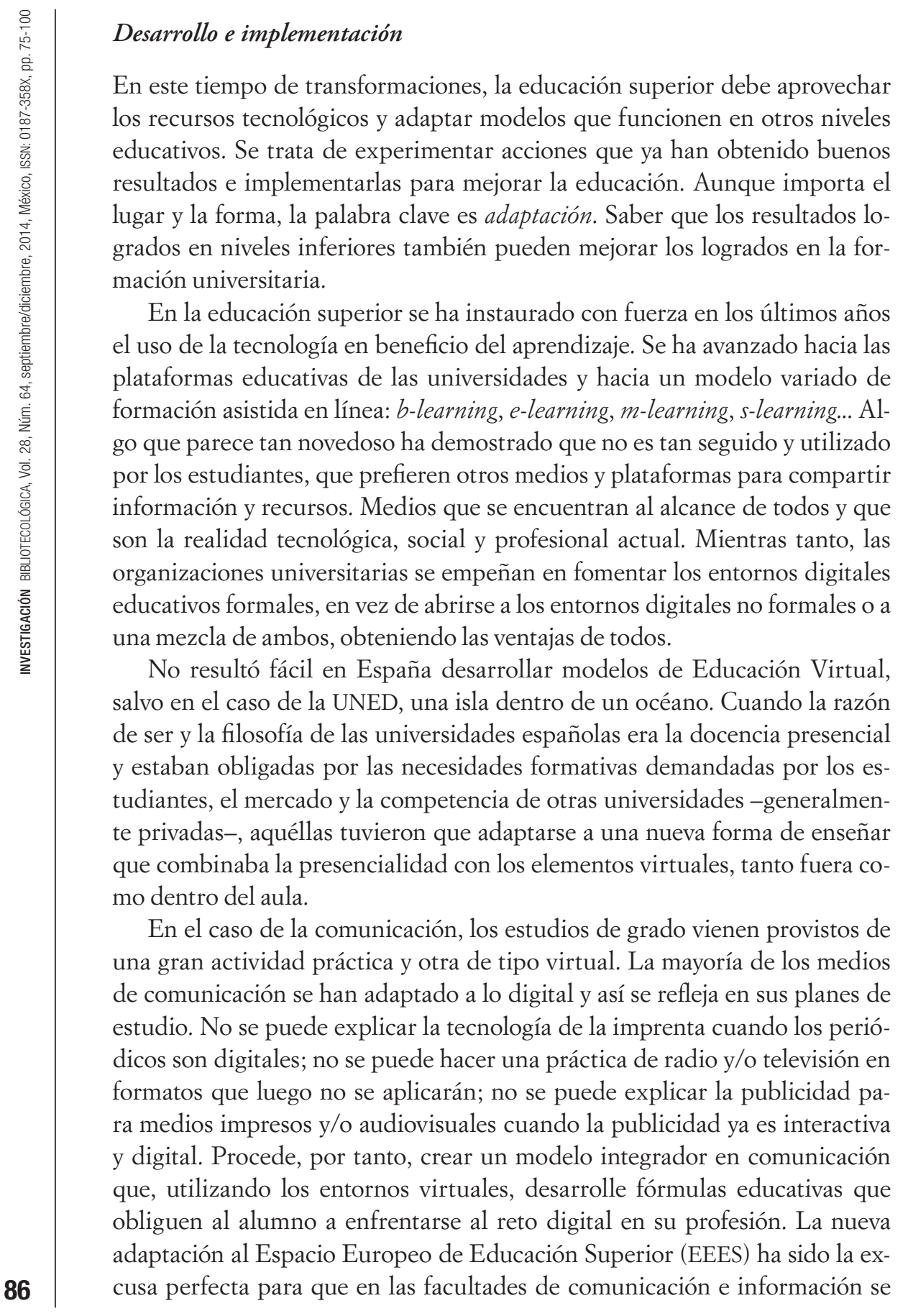


propongan nuevas fórmulas para hacer que estos estudios ofrezcan propuestas más dinámicas y, sobre todo, un mayor uso de la tecnología actual, la misma que se aplica en el ámbito profesional y en sus prácticas. Si bien el modelo teórico ha sufrido una menor adaptación, los casos prácticos, los trabajos en laboratorios y los estudios de radio y televisión son una muestra más de las necesidades de desarrollo e implementación hacia una formación digital de los estudiantes de comunicación.

\section{Aplicaciones prácticas}

Algunas universidades han experimentado diversas plataformas (WebCT, Blackboard, Sakai, Dokeos, Moodle) en busca de una opción eficaz y barata. Algunas de ellas han considerado fundamental utilizar una que no sea de pago, al menos en situaciones como la actual, donde los recursos son escasos, especialmente los económicos. En ese sentido, los docentes y los alumnos han ido saltando de una plataforma a otra sin tener claro cuál era el beneficio de usar esta tecnología.

En el caso de las asignaturas de periodismo y/o publicidad y relaciones públicas, como es Documentación Informativa, desde hace cuatro cursos se ha adaptado la asignatura a Moodle, ya que las experiencias con WebCT no fueron especialmente participativas para el alumno. Se recogen, además de los apuntes y textos del profesor, campañas de publicidad para que el alumno participe y aprenda de forma activa, y también se facilitan recursos y fuentes documentales para el normal desarrollo de las prácticas. El resultado por parte del alumno se plasma en el sitio de prácticas. Todas estas actividades son respondidas por el profesor dentro de la plataforma y a su vez el alumno debe volver a revisar lo que el tutor le ha recomendado; pero esto no ha resultado ser suficiente para el aprendizaje.

Es la parte práctica el mejor exponente de un tipo de educación que en el caso de los medios digitales requiere de herramientas que la hagan posible, como ha sido el portal Publidocnet creado por y para estudiantes (http:// www.publidocnet.com/), que es un Centro Documental para la Conservación del Patrimonio Publicitario Español (Figura 4). Crear publicidad para Internet sólo se puede hacer dentro de Internet, de ahí que algunas asignaturas no tienen razón de ser alejadas de plataformas virtuales y usando las ventajas de las redes sociales, como Facebook y especialmente Twitter.

Los entornos de comunicación y relación que Internet brinda otorgan al docente la posibilidad de convertirse en un community education manager, es decir, en un gestor de la comunidad educativa que lidera apoyado por su grupo de estudiantes. Se da por tanto un sistema global de aprendizaje en la 


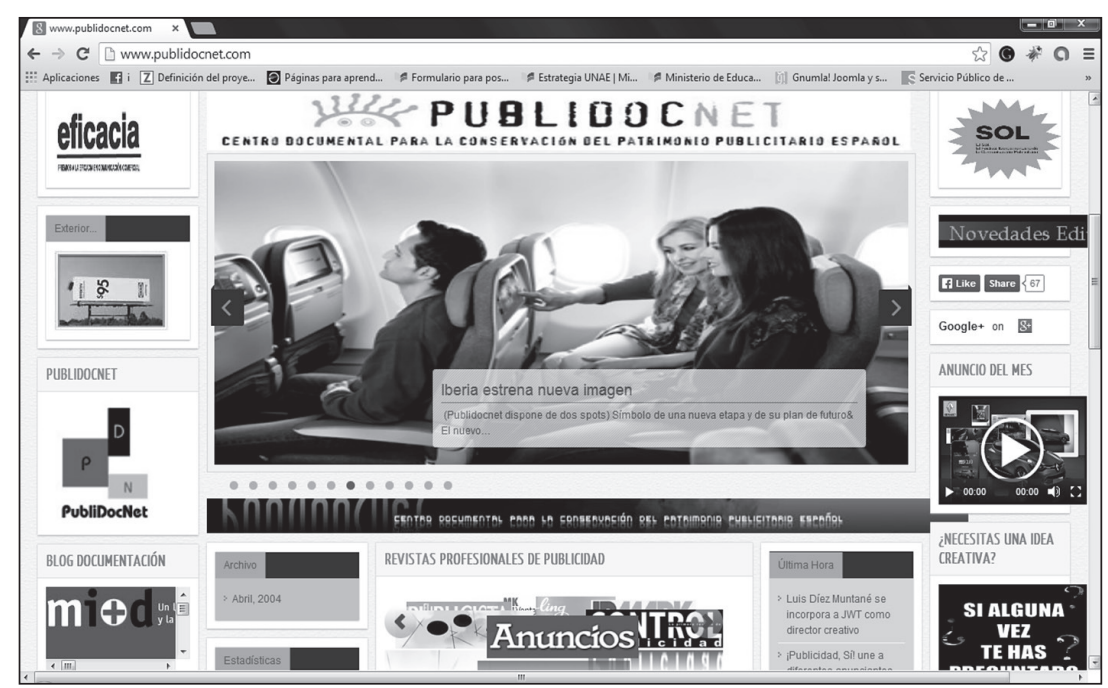

Figura 4. Portal de prácticas reales en Publidocnet.

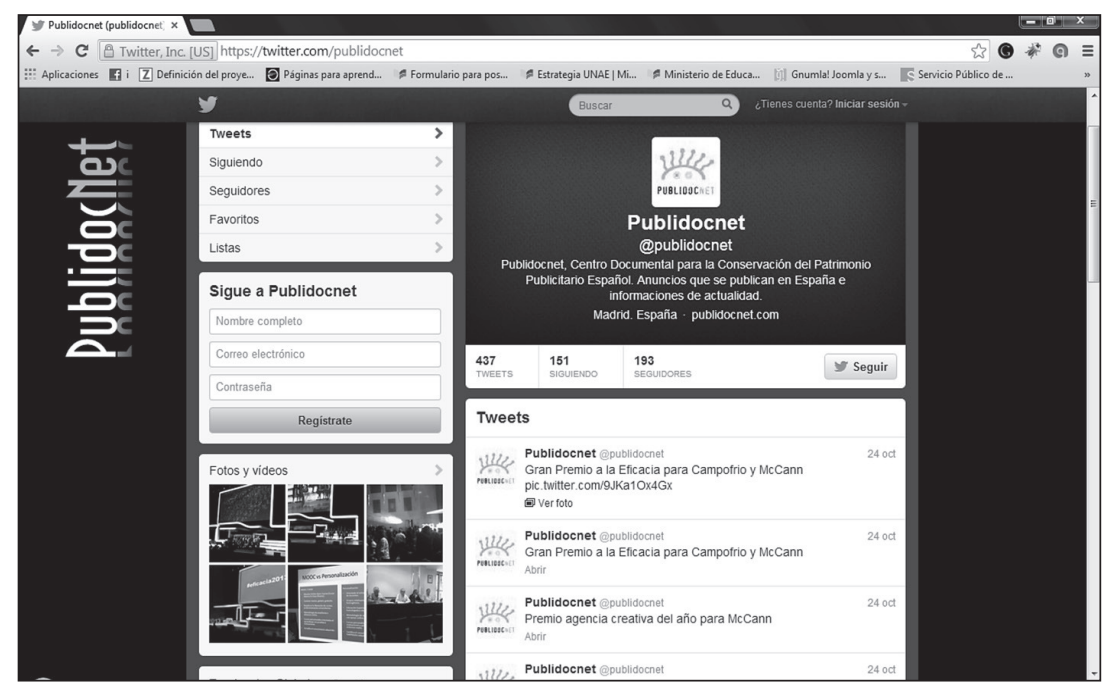

Figura 5. Perfil de Publidocnet en Twitter.

Nube o Cloud Learning (c-learning), teniendo como base a un docente como gestor de la comunidad educativa, que emplea tanto los espacios digitales formales (los propios que le ofrece la organización educativa) como los no formales (los que están en la actualidad en la red y que los estudiantes utilizan a diario). En este sentido, Facebook o Twitter (Figura 5) pueden ser utilizados como instrumentos colaborativos a favor de la educación, además 
de como ejemplo y herramienta para aprender de manera práctica las habilidades de comunicación que se utilizan el día de hoy en la empresa privada, pero sin ser los únicos.

Se trata de ubicar al alumno en su entorno habitual, en el que más cómodo se encuentra, pero no conoce desde el punto de vista de su uso profesional. Los estudiantes no necesitan entrenamientos adicionales, ya que conocen el sistema a la perfección. Por ejemplo, el modelo de página o de grupo que tiene Facebook es óptimo para impartir una formación dinámica, desde la cual es posible afiliar al alumnado. La página y el grupo poseen mayor número de herramientas frente al perfil, ya que se trata de un entorno administrable por varios usuarios (profesores en este caso) y ofrece datos estadísticos útiles para evaluar el impacto de la formación, justo como en plataformas educativas tipo Moodle.

El apartado para la información del grupo de Facebook es perfecto para incluir el programa y el cronograma del curso, los datos de contacto del profesorado, el personal auxiliar y de apoyo, el horario de tutorías, normativas, etc. El muro sería la zona de intercambio y encuentro común de estudiantes y docentes, donde se comparten contenidos formativos (textos, videos), preguntas, opiniones, aportaciones adicionales, etc. Los grupos poseen un apartado para enlaces, fotos, videos y audios para compartir los materiales didácticos de apoyo del programa. Las herramientas de comunicación síncrona como el chat se emplearían para las tutorías; mientras que el foro sería para los debates programados. Las herramientas de comunicación asíncronas, como el correo, son ideales para las tutorías y el intercambio más personal y privado.

Los eventos informan sobre los acontecimientos del curso (fechas de encuentros presenciales, foros, tutorías) a nivel formal, pero también a nivel informal. Las notas son herramientas que, al ser de uso colectivo y público, son perfectas para hacer las funciones de wikis para el trabajo en grupo y convierten las respuestas en base de conocimiento. El propio muro ya es una gran wiki de 420 caracteres.

De todas ellas, quizás sea Twitter la que tiene más posibilidades, especialmente para los futuros profesionales de la comunicación. Así, se pide a los alumnos que utilicen esta herramienta para crear textos cortos, llenos de fuerza informativa, y a la vez que los hagan visibles en una comunidad o red virtual, dejando de lado el uso lúdico y para ocio al que están acostumbrados. El mismo modelo serviría para quienes practican la publicidad, pues han de ensayar modelos de publicidad digital capaces de conseguir la atención del consumidor en apenas unos segundos. Pero serían también fuente informativa y lugar para conocer y relacionarse con las empresas de la comunicación y sus expertos. La comunicación actual tiene más fuerza en la 
medida en que los estudios dispongan de clases prácticas y se implique a los alumnos para que desarrollen su creatividad proyectando prácticas, como campañas, y la hagan extensibles a las redes sociales.

Los autores de este artículo han puesto en marcha estas ideas de manera práctica durante el curso 2011/12, en el proyecto titulado "Portales de apoyo al campus virtual creados para el desarrollo de recursos y prácticas colectivas" y en el curso 2012/13 "Uso de dispositivos inteligentes (tablets, smartphones...) como herramientas educativas en el campus virtual y portales educativos (Publidocnet): aplicaciones prácticas en la asignatura Documentación Informativa", dentro del marco de "Proyectos de Innovación y Mejora la Calidad Docente" promovido por el Vicerrectorado de Evaluación de la Calidad de la Universidad Complutense de Madrid. En las asignaturas de publicidad informativa, documentación publicitaria y documentación informativa se han creado con y para los alumnos una página en Facebook (http:// www.facebook.com/publidocnet), un canal de Twitter (http://twitter.com/ publidocnet) y se ha mejorado el portal PublidocNet (http://www.publidoc net.com), en los cuales se han perseguido los objetivos anteriormente citados.

\section{Evaluación del modelo}

Cualquier actividad educativa tiene un valor mayor cuando se somete a una evaluación, pero si además evalúan empresas ajenas, ese valor es doble. Actualmente no hay empresa, organismo o institución que no esté sometido a un control y/o evaluación. En el ámbito educativo también sucede de este modo. Este trabajo propone evaluar cada una de las actividades educativas por empresas especializadas, pero también por los propios usuarios, los alumnos.

Siguiendo el ejemplo propuesto, School of One es un método docente que ha sido evaluado con éxito y que constituye una forma de enseñar que recupera la metodología de los tiempos de Platón y Aristóteles, en los que un maestro se rodeaba de los mejores alumnos y conseguía obtener buenos resultados de cada uno de ellos. El grupo de estudiantes ha crecido significativamente, de ahí la importancia de plantear una asignatura personalizada (cuanto más mejor), aunque eso suponga una carga de trabajo extra para profesores y alumnos.

Por último, pocas instituciones educativas evalúan a largo plazo la eficacia de la formación y del éxito de sus planes de estudios. Y esto es un procedimiento fundamental para formar profesionales válidos para lo que el dinámico mundo empresarial demanda. Se trataría de conocer en los años posteriores a finalizar los estudios de los alumnos cuál ha sido el grado de experiencia y de inserción en el mercado laboral. Para mejorar hay que hacer 
encuestas a alumnos durante los cinco años siguientes, tanto a quienes están trabajando profesionalmente como a quienes se desempeñan en otra especialidad. La evaluación supone recursos financieros y personales, pero cuando se hace bien garantiza a la institución educativa mejores resultados, pues se detectan carencias que se pueden corregir y mejorar. También en los sondeos a empresarios y directores de recursos humanos de empresas de comunicación se podría precisar si las capacidades impartidas están alineadas con las necesidades que la sociedad demanda.

En lo que se refiere al proyecto Publidocnet, son muchos los alumnos que una vez terminados sus estudios han continuado colaborando, y también dentro de su vida profesional. Igualmente ha sucedido con profesionales que han conocido el proyecto y han participado de manera activa, lo que supone una interacción y transferencia de conocimientos y habilidades desde el mundo académico y del empresarial. También sirve esto como piedra de toque para conocer la evolución de los antiguos estudiantes a través de sus experiencias y participaciones.

\section{De ALUMNO A EMPRENDEDOR: ¿CÓMO CONSTRUIR PUBLICACIONES?}

Si se aplican métodos docentes parecidos a School of One o como los aplicados en los proyectos de estos últimos cursos en nuestros proyectos, el alumno aprenderá a crear sus propias empresas, a poner sus publicaciones en comunicación y/o su publicidad en los medios digitales. Veamos un ejemplo:

Cada año, en invierno, tengo el gusto de dar una conferencia en el auditorio de La Cartuja de Sevilla a un millar de alumnos de Económicas de diferentes facultades andaluzas. Suelen ser de último curso. Cada año, a mitad de la charla, formulo la misma pregunta: “¿Cuántos de vosotros tiene previsto emprender alguna cosa, proyecto, negocio o lo que sea en los próximos cinco años?”. La respuesta cada año va a peor. Normalmente apenas una decena levanta la mano. Luego vuelvo a inquirir: "Entonces, ¿cuántos queréis ser funcionarios?”. Los brazos en alto aumentan, pero tampoco puedo decir que crezca un bosque frente a mí. [...] Ellos no tienen la culpa, la culpa es del entorno que hemos fabricado y que adormece el deseo de ponerse en marcha. (Vidal, 2010: 12)

Atendiendo a las palabras de Marc Vidal, cabría decir que hay algo que no está funcionando en la universidad, al menos en la española. No sólo dibuja un panorama poco alentador, que no promueve el espíritu emprendedor o el autoempleo, sino que tampoco es visto como útil a la hora de afrontar 
el mercado laboral donde los estudiantes no ven futuro. Haciéndose eco de varios informes, el periódico Expansión comenta en un artículo titulado “¿Dónde quieren trabajar los universitarios?”:

La visión de los estudiantes sobre la formación que reciben en sus universidades también es crítica en términos de inserción laboral. Según el estudio del instituto alemán Trendende, el $40 \%$ de los alumnos españoles cree que la universidad no les proporciona las habilidades necesarias para el mercado de trabajo. [...] En el caso de los alumnos nacionales de titulaciones relacionadas con el mundo empresarial, [...] un importante $40 \%$ piensa que no se les proporcionan las competencias requeridas en el desempeño profesional. (Rodríguez, 2011: s. pág.)

Es clave, entonces, atender y seguir las conclusiones para el Diseño de Políticas Públicas, para los Educadores y para la Implementación Práctica que propone el estudio del GEM (Global Entrepreneurship Monitor) coordinado por Alicia Coduras, titulado "GEM Special Report: Una perspectiva global sobre la Educación y Formación emprendedora”. En él donde se destaca lo siguiente:

1. Los resultados indican que los individuos tienden a recibir formación a la hora de emprender como parte formal de su educación. Esto demuestra la importancia que tienen las escuelas, institutos y universidades en cuanto a establecer una base para el emprendimiento. [...] se podría animar a los educadores de primaria y secundaria para que incluyesen programas efectivos de formación dentro de sus planes de estudios.

2. El descubrimiento de que la formación formal e informal se solapan implica que la formación formal puede proporcionar una base, pero que muchas personas necesitan conocimientos y habilidades específicas, quizás cuando empiezan a interesarse en emprender o ya han comenzado a hacerlo. Fuentes rentables de formación, como el auto estudio y programas basados en Webs de Internet [...]

3. La formación emprendedora en el nivel terciario no debería estar limitada a aquellos que tengan objetivos empresariales. Esto permitiría que el emprendimiento fuese una opción profesional para todo el mundo y que puede ser ejercida cuando una combinación de circunstancias la convierta en una opción viable. (Coduras et al., 2010: 52)

No obstante, hay luz al final del túnel. Según el informe GEM España:

En España, la educación emprendedora está más desarrollada en la etapa posterior a la escolar, si bien, existen programas excelentes en formación para escolares entre los que destacan los de Valnalón, cuyo centro tecnológico y de desarrollo 
del emprendimiento los aplica, no sólo en Asturias, sino en otras regiones españolas. Por consiguiente, lo que falta en nuestro país no son ideas ni materiales didácticos, sino profesorado preparado para impartirlos y una amplia cobertura del ámbito escolar en cuanto a la implantación de esta materia por todo el estado. (GEM Spain, 2010: 158)

\begin{tabular}{|l|c|l|c|}
\hline \multicolumn{4}{|c|}{ Formación para emprender en la población activa según nivel de estudios } \\
\hline$\%$ sin estudios & $0.0 \%$ & $\%$ sin estudios 2009 & $12.1 \%$ \\
\hline$\%$ primaria 2008 & $16.9 \%$ & $\%$ primaria 2009 & $21.4 \%$ \\
\hline$\%$ secundaria 2008 & $22.3 \%$ & $\%$ secundaria 2009 & $31.5 \%$ \\
\hline$\%$ medios y F.P. 2008 & $26.0 \%$ & $\%$ medios y F.P. 2009 & $42.0 \%$ \\
\hline$\%$ superiores 2008 & $24.7 \%$ & $\%$ superiores 2009 & $44.1 \%$ \\
\hline
\end{tabular}

Figura 6. Principales indicadores de prevalencia de la formación para emprender en la población activa española. (GEM Spain, 2010: 159)

Se puede observar en la Figura 6 que, afortunadamente, la tasa de formación para el emprendimiento o prevalencia en formación para emprender ha ascendido de manera considerable en la población activa española en los últimos años. Este hecho está relacionado con el Informe GEM sobre España 2010 con la intensificación de la enseñanza tras el periodo escolar en materia de emprendimiento. Iniciativas como Valnalón (http://www.valnalon.com/ valnalon_educa/) son las promotoras de la mejoría de los datos, los cuales consideran fundamental el aprendizaje de habilidades innatas emprendedoras ya desde la escuela. Pero este desarrollo no termina aquí, ya que dicho proyecto educativo está estructurado a modo de cadena cuyo primer eslabón es la Educación Primaria y el último es la Formación Profesional o la universidad.

De igual modo, como muestra la Figura 7, no existe una clara y directa relación entre nivel de estudios y actividad emprendedora, si bien se atiende

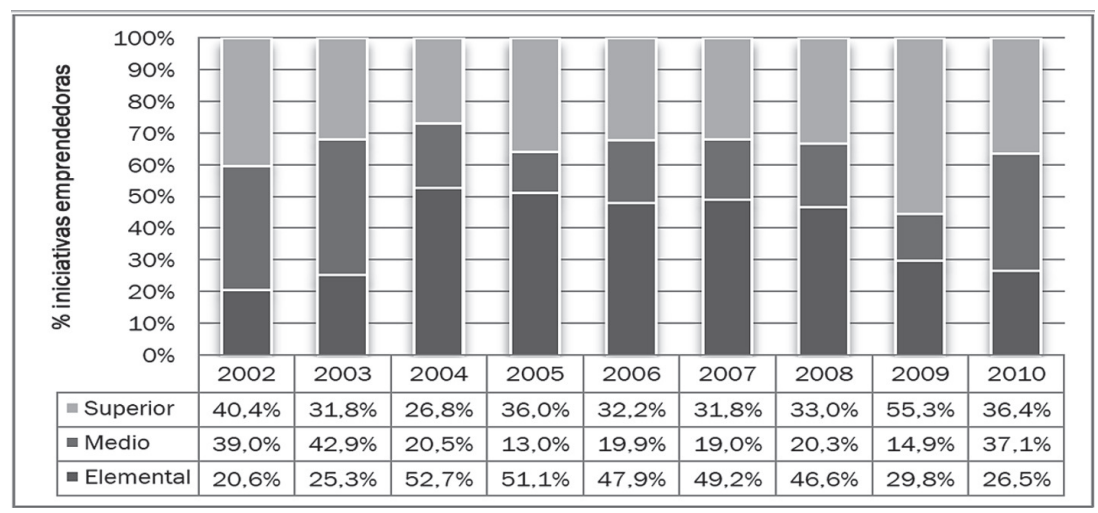

Figura 7. Evolución de la distribución de la actividad emprendedora total, en función del nivel educacional de los emprendedores (2002-2010). (GEM Spain, 2011: 34) 
mucho más a condicionamientos socioeconómicos de carácter nacional e internacional. Atendiendo a los niveles educativos la Tasa de Actividad Emprendedora (TEA) indica "[...] que es proporcionalmente más elevada entre los que tienen estudios superiores”. (GEM Spain, 2012: 46)

¿Pero qué hacen las Escuelas de Negocios para que sean cuna del emprendimiento y no tanto las universidades, pese a poseer los canales de I+ D+i (investigación, desarrollo e innovación)? ¿Cuál es el objetivo actitudinal que pretenden imprimir las Escuelas de Negocio a sus estudiantes? A finales de mayo de 2011, la Escuela de Organización Industrial (EOI) trató el tema en su Mediateca, donde incluyó entrevistas a profesores y empresarios bajo el título "¿El empleado del futuro? Capacidades y Habilidades”. Todos coinciden en que el empleado del futuro tiene que ser emprendedor (aunque sea dentro de una organización), innovador y flexible. Un conocedor de la tecnología, especialmente de Internet, la Web 2.0 y las Redes Sociales. Debe tener capacidad de aprender, de liderazgo, de mantenerse al día en el uso de las Nuevas Tecnologías, de entender el contexto general y algo absolutamente esencial: poder interactuar con otros individuos, con otros paisajes, con otros entornos, con otras culturas, con otros países, etcétera.

Matylda Czarnecka (2011), en Poynter, ha detectado que los periodistas de los últimos tiempos tienen iniciativas emprendedoras, animadas por las facilidades de las Nuevas Tecnologías, cuyas herramientas más directas son los blogs, pero también las Redes Sociales, especialmente Twitter y Facebook. Hablar en la actualidad de Comunicación es lo mismo que hacerlo de Marketing, Entornos Digitales, Documentación, Publicidad, Periodismo, Imagen, Audiovisual e Información, pero también de Nuevas Tecnologías e Innovación y, por supuesto, de autoempleo y de emprendimiento. En este sentido, los estudiantes participantes de los proyectos de estos dos últimos años presentaban un índice de uso de blog de $19 \%$ al inicio del curso, mientras que al final esta cifra era de $53 \%$, y se había incrementado también la tasa de blogs colaborativos, en los que se creaba más información de la que se citaba. Por otro lado, el uso que le daban inicialmente al blog era lúdico y de ocio, mientras que al final del curso las temáticas se ampliaron y los usos fueron más profesionales.

Los profesionales de la comunicación son especialistas en los temas que tratan. Para cualquier empresa es esencial conocer el mercado y el público, detectar las tendencias y orientarse sobre ellas. También son expertos en la obtención de recursos, habilidad necesaria para cualquier emprendedor; son buenos investigadores con gran capacidad para relacionarse, por lo que emprender implica trabajo de grupo y comunicación tanto externa como inter- 
Por su parte, Marshall McLuhan, en su libro Comprender los medios de comunicación: las extensiones del ser bumano, afirma que un medio sustituye a otro, haciéndose tan atractivo que todo pasa por él, incluida la formación, como es ahora el caso de Internet y la Web 2.0 y 3.0. Es decir, Internet y las Nuevas Tecnologías son el medio para entrenar y fomentar las habilidades del emprendimiento en los estudiantes universitarios.

José Luis Orihuela define el beneficio del uso de las Nuevas Tecnologías a través de Twitter, comentando que es útil para las organizaciones en los aspectos siguientes:

Atención al cliente, Branding, Comunicación externa, Comunicación interna, Difusión y cobertura de eventos, Empleo, Gestión de conocimiento, Gestión de crisis, Gestión de proyectos, Gestión de la reputación, Innovación, Investigación, Marketing, Monitorización, Networking, ofertas y promociones, relaciones con la prensa, relaciones públicas, tráfico hacia el sitio web, ventas y visibilidad. (Orihuela, 2011: 80-82)

Del mismo modo, cuando Javier Celaya responde a la pregunta "¿Qué puede hacer una empresa en las redes sociales?” (2011: 155) mencionando las acciones siguientes: "creación de un perfil corporativo [...], creación de un grupo de interés [...], segmentación del mercado [...], realización de encuestas [...], análisis del comportamiento del usuario [...], atención al cliente [...], publicidad on-line [...] y escuchar las conversaciones" (2011: 156-159), está relatando las habilidades que puede llevar a cabo cualquier profesional de la Comunicación con conocimiento en redes sociales.

Esta idea es más que suficiente para entender que utilizar las redes sociales y/o ser bloguero, durante la educación superior o incluso antes, puede aportar a los estudiantes la capacitación adecuada para desarrollar su labor como periodistas y empresarios digitales. Existen muchos casos dentro de la profesión periodística de algunos que comenzaron como blogueros y posteriormente fueron contratados por los grandes periódicos y compañías de prestigio.

En nuestro proyecto detectamos que sólo $9 \%$ de los estudiantes no disponía de cuenta en Twitter; al final del curso el $100 \%$ contaba con una. Además, la tendencia del uso de los alumnos se había trasladado de ser de ocio y opinión (41 \%) a ser mucho más profesional (82 \%), siguiendo una temática más concreta e interactuando con profesionales de dicho sector, obteniendo incluso que muchos los siguieran. Durante las prácticas, el objetivo fue mostrar los perfiles de profesionales y empresas de publicidad más destacados y el modo de interactuar con ellos, al aprender los estudiantes un uso profesional de esta herramienta. 
Las capacidades de emprender también se desarrollan gracias a la práctica con herramientas como blogs y Facebook, al seguimiento de consejos e instrucciones como los publicados en Facebook + Journalism (http://www. facebook.com/journalists) o Twitter for Newsrooms (La guía de Twitter para las redacciones, disponible en http://media.twitter.com/newsrooms), o bien creando una revista electrónica en PDF y colocándola en ISSUU. Esto obliga a los estudiantes a buscar y conseguir un público, establecer una línea editorial que genere un número suficiente de lectores y resolver tareas de actualización y gestión de los contenidos; además, deben ocuparse de la generación de un proceso editorial, del uso de diversos medios para los contenidos (video, texto...), del compromiso con su público, del control de los canales de distribución, de la selección de una plataforma y de los problemas de migración, así como de la selección y el cambio de un modelo de negocio, el posicionamiento y el marketing; la gestión de recursos, la optimización y la gestión del tiempo, el trabajo con fuentes y, lo más importante, la monetización de su trabajo, la gestión financiera de su inversión y el RSI (Retorno Sobre la Inversión, en inglés ROI-Return on Investment) de la misma.

Se trata de aprender a elaborar un plan de negocio, pero es más importante llevarlo a cabo y ponerlo en marcha, readaptándolo y orientándolo según las circunstancias, con el objetivo de conseguir la satisfacción como emprendedor, el desarrollo de una acción profesional y la obtención del beneficio que es el fin principal del emprendimiento. La parte decisiva es la puesta en común de las lecciones aprendidas y el hecho de que los proyectos activos no se queden tan sólo en el aula, sino que se presenten a concursos de emprendedores para seguir evaluando si son viables y sostenibles. Se trata de llevar la práctica universitaria a la realidad profesional. O mejor, que no sean en realidad prácticas, sino que se consideren proyectos que buscan la profesionalización y monetización de las clases.

La educación en general se tiene que convertir en un espacio de aprendizaje para el desarrollo de I+E (Innovación y Emprendimiento), donde se compartan las propuestas entre estudiantes y donde todos puedan aprender también de sus pares. Donde iniciativas como la NewU: News Entrepreneurs Working Through UNITY, programa para periodistas que quieren convertirse en emprendedores, que se creó en 2009 en EUA, puedan formarse dentro del ámbito universitario o antes, dentro del proceso educativo.

Existe la necesidad de enseñar cómo emprender, pero no sirve de nada sin una cultura financiera adecuada. Es posible que las iniciativas de $\mathrm{I}+\mathrm{D}+\mathrm{i}$ que se desarrollan en la universidad no tengan la capacidad de dar el salto, no sólo por falta de cultura emprendedora sino también por falta de cultura financiera. Obtener los recursos económicos de un proyecto de $\mathrm{I}+\mathrm{D}+\mathrm{i}$ re- 
quiere un alto nivel de conocimientos en financiación y administración de recursos económicos, pero también de gestión de intangibles, como son los de personal y tecnología. Todo ello es necesario para los estudios universitarios en cualquier carrera, pero especialmente en las de Comunicación, debido a las altas posibilidades que tiene este sector gracias al boom tecnológico. Cuando se habla de financiación se requiere saber gestionar créditos, conocer hasta dónde podemos llegar con los Bussiness Angels, grupos de inversión, crear planes de riesgo... Y lo que es más importante, hay que conocer cómo funciona la gestión de los recursos económico-financieros. En este sentido, los estudiantes han conocido el costo de la plataforma para el portal Publidocnet, valorando económicamente el número de horas dedicadas y simulando el ROI en publicidad de dicho portal para conocer el beneficio que se hubiera obtenido.

El llamado Plan de Bolonia retoma el espíritu multicultural de aprendizaje de idiomas y culturas, de entornos y grupos de trabajo con estudiantes de los países de la UE, que se ha estado fomentando desde que surgieron las Becas Erasmus y Socráctes/ECTS (European Credit Transfer and Accumulation System-Sistema Europeo de Transferencia y Acumulación de Créditos), con el fin de generar un espacio económico y profesional común para toda Europa. En la actualidad, las Nuevas Tecnologías permiten la generación de dichos entornos sin la necesidad de viajar, así como la creación de un único espacio de educación superior donde los alumnos de Europa pueden cursar asignaturas de otras universidades.

Pero esto hay que promocionarlo desde la escuela primaria hasta la educación superior. Un mundo global exige un profesional o emprendedor con visión, una visión general de su sector, con un carácter internacional que le permita que el mundo entero sea el ámbito para ejercer su profesión y/o que su propia empresa tenga una visión internacional o con capacidad de internacionalizarse llegado el caso. Estas tecnologías aplicadas a la comunicación son justamente lo que permite que el ámbito sea internacional, al estilo de WikiLeaks. En tal contexto, ¿el idioma sería una barrera? No en realidad, ya que el fomento de la educación con entornos multiculturales desde la educación primaria ofrece el conocimiento de otros idiomas de manera sencilla y natural.

Del mismo modo, el fomento de asignaturas optativas y compartidas por varias licenciaturas de una misma universidad o universidades de una Región o Comunidad Autónoma, como el Proyecto ADA-Madrid (Proyecto Aula a Distancia y Abierta de la Comunidad de Madrid, http://moodle.upm.es/adamadrid/) no es más que otra manera de fomentar el trabajo multidisciplinar, otra de las características del profesional y/o emprendedor de futuro, o sería más justo decir del presente. 
La educación actual se tiene que desarrollar en entornos multidisciplinares, multiculturales, multilingüísticos e internacionalizados; esto ahora es posible gracias a las tecnologías actuales. La economía digital, que es la economía actual, demanda profesionales y emprendedores con estas características:

Quizá, la manera de realizar una labor profesional pase en la actualidad por el autoempleo y emprender, gracias a las oportunidades que aporta la tecnología y las nuevas profesiones que en términos de comunicación digital y elaboración de contenidos están surgiendo. Si bien, emprender dijimos que entraña un riesgo y mucho más en España, la ilusión y la pasión con que se ejerce por tradición la profesión de informador y comunicador, permite superar cualquier posible barrera. (Marcos \& Alcolado, 2011: 16)

Ese es uno de los retos educativos en el que instituciones, docentes y alumnos habrán de cambiar la forma de enfrentarse a su futuro profesional, aceptando las propuestas de mejora en los estudios, especialmente en Comunicación.

\section{Conclusiones}

Del modelo propuesto hemos de deducir que la mejor alternativa para una educación de calidad es personalizar o reducir el colectivo a grupos pequeños. Pero ése es sólo el primer paso, pues algunas universidades privadas en España ya cuentan con ese tipo de grupos y no por eso han conseguido mejoras en sus resultados. El verdadero valor es el esfuerzo individual de cada estudiante sumado con el del profesor, que deberá conocer las capacidades de cada alumno y hacer que avance, frente a otros que han de llevar un ritmo diferente. Si colocas en el mismo espacio físico estudiantes avanzados con otros de inferior categoría, el problema es que los primeros perderán, pues tratarán de adaptarse al ritmo de los segundos. En nuestro modelo cada alumno avanza en función de sus capacidades y el profesor es un apoyo.

La universidad en particular, pero también toda la cadena educativa desde primaria, se tienen que convertir en entornos I+E (Innovación y Emprendimiento) para el desarrollo sano y natural de las habilidades de los estudiantes y para cumplir con los objetivos que la sociedad demanda de la educación. La comunidad docente se tiene que reinventar para favorecer y motivar a los estudiantes y dinamizar las habilidades que favorecen su creatividad y emprendimiento.

El uso de las Nuevas Tecnologías de entornos educativos formales y no formales, que hemos denominado cloud learning (c-learning), permite un 
aprendizaje multiorganizacional entre países en el cual se fomentan los grupos de estudio multidisciplinares, multiculturales, multilingüísticos e internacionalizados, características que los profesores, como profesionales, deben promover. Asimismo, el profesor contribuye asumiendo el rol de community education manager, lo que facilita la educación personalizada y el fomento de la cultura del emprendimiento.

No es tiempo de quedarse quieto, sino de innovar. Las opciones son varias e interesantes, abiertas a la participación de los alumnos y a una mayor relación entre los docentes, quienes han dejado su mundo aislado para compartir recursos, maneras de enseñar y, sobre todo, puntos de acción en común. La educación del siglo XXI es una suma de muchas personas y voluntades de una mismo área y de otras colaterales; de instituciones educativas que quieren mejorar sus resultados y de personas (profesores y alumnos) que desean mayor conocimiento que el que pueden conseguir en las redes sociales. También que el docente ayude a los alumnos a entender, valorar e interpretar esos datos que manejan en cada clase.

\section{Bibliografía}

Bohn, R. E. \& Short, J. E. (2009). How Much Information? 2009. Report on American Consumers. California: University of California. Disponible en: http://hmi.ucsd.edu/pdf/HMI_2009_ConsumerReport_Dec9_2009.pdf [Fecha de consulta: 11-11-2012].

Coduras, A.; Levie, J.; Kelley, D.; Saemundsson, R. \& Schoot, T. (2010). Global Entrepreneurship Monitor Special Report: A Global Perspective on Entrepreneurship Education and Training. Babson College, Universidad del Desarrollo, Reykiavik University.

Carr, N. (2011). Superficiales. ¿Qué está haciendo Internet con nuestras mentes? Madrid, Taurus.

Celaya, J. (2011). La empresa en la web 2.0. El impacto de las redes sociales y las nuevas formas de comunicación online en la estrategia empresarial. Madrid, Gestión 2000.

Czarnecka, M. (2011). "7 razones por las que los periodistas son buenos emprendedores", en Poynter. Disponible en: http://www.poynter. org/how-tos/leadership-management/134757/7-reasons-why-jour nalists-make-good-entrepreneurs/ [Fecha de consulta: 14-07-2012].

Escuela de Organización Industrial EOI (2011). ¿El empleado del futuro? Capacidades y Habilidades. Madrid, EOI. Disponible en: http:// www.eoi.es/mediateca/video.php?videoid $=876 \&$ PHPSESSID $=$ 52499d67ce95a2d1e647aeec11f5b700 [Fecha de consulta: 22-022012]. 
García, I.; Peña-López, I.; Johnson, L.; Smith, R.; Levine, A. \& Haywood, K. (2010). Informe Horizon: Edición Iberoamericana 2010. Austin, Texas, The New Media Consortium.

GEM Spain (2010). Global Entrepreneurship Monitor Spain: Informe Ejecutivo 2009.

- (2011). Global Entrepreneurship Monitor Spain: Informe Ejecutivo 2010 .

- (2012). Global Entrepreneurship Monitor Spain: Informe Ejecutivo 2011 .

Marcos Recio, J. C. \& Alcolado Santos, J. (2011). A New Educational Paradigm: from e-learning to cloud learning (c-learning). Knowledge in the Cloud. EDULEARN11, $3^{\text {a }}$ Conferencia Internacional de Educación y Nuevas Tecnologías para el Aprendizaje. Barcelona (España). 4, 5 y 6 de julio 2011.

(2012). "Profesionales de la comunicación y espacios de empleo en entornos digitales", en Competencias interdisciplinares para la comunicación y la información en la sociedad digital. Madrid, ICONO 14.

- (2013). Publidocnet. Recursos para la formación virtual a través de los medios de comunicación. Madrid, Fragua.

Ingenieros.es (2013), "Once países europeos lanzan los primeros cursos masivos abiertos online a nivel universitario", en Ingenieros.es Portal de ingenieros españoles, 24-04-2013. Disponible en: http:// www.ingenieros.es/noticias/ver/once-paises-europeos-lanzan-losprimeros-cursos-masivos-abiertos-online-a-nivel-universitario/3571 [Fecha de consulta: 21-10-2013]

Orihuela, J. L. (2011). Mundo Twitter. Madrid, Alienta Editorial.

Rodríguez, E. (2011). “¿Dónde quieren trabajar los universitarios?”, en Expansión, 28-01-2011, Madrid. Disponible en: http://www. expansion.com/2011/01/28/empleo/mercado-laboral/1296240189. html?a $=$ e6c1c8732e5ada61ae679315a98fda02\&t=1308504784 [Fecha de consulta: 01-07-2012]

The Economist (2011). "The leaky corporation”. Feb 24th 2011. Disponible en: http://www.economist.com/node/18226961 [Fecha de consulta: 01-07-2012]

Vidal, M. (2010). Contra la cultura del subsidio. La Microburguesía low cost y su escasa iniciativa empresarial. Madrid, Gestión 2000. 